risk factors independent of the individual's own SES, but the mechanisms have not fully been understood. Our aim was to assess the association of neighbourhood and individual SES with cardiovascular risk factors in an Eastern German population with exceptionally high cardiovascular mortality and unemployment rates.

Methods We used cross-sectional data of 1779 inhabitants of the city of Halle (Saale), aged 45-83 years, who participated in the population-based CARLA study. We calculated linear mixed models to assess the age-adjusted influence of neighbourhood SES (defined as neighbourhood-specific unemployment rates for 39 administrative districts of the city) and individual SES (defined as number of education years) on smoking (defined as number of currently smoked cigarettes/day), systolic blood pressure (SBP), and body mass index (BMI). Spatial dependencies within and between neighbourhoods were adjusted for by using ICAR models.

Results The unemployment rate ranged from 6.3 to $35.3 \%$ between neighbourhoods. For smoking, there was a statistically significant increase of 0.11 cigarettes smoked/day per $1 \%$ increase in the neighbourhood's unemployment rate in men ( $95 \%$ CI 0.09 to 0.12 ), and a decrease of 0.59 per increase in education years (CI -0.62 to -0.56 ), but a weaker association in women (regression coefficients ( $\beta$ ) for unemployment rate and education years 0.054 (CI 0.039 to 0.067 ), and -0.21 (CI -0.24 to -0.19$)$ ). There was no statistically significant association of SBP with SES in men ( $\beta=-0.07$ (CI -0.22 to 0.08 ) for unemployment rate, and -0.15 (CI -0.69 to 0.38 ) for education years), while in women, there was a statistically significant decrease in SBP of $0.79 \mathrm{mmHg}$ per increase in education years (CI -0.82 to -0.76$)$, and an increase with unemployment rate ( $\beta=0.04$, CI 0.03 to 0.06 ). BMI was statistically significantly associated with education in men and women (0.11 decrease in BMI per increase in education years in men (CI -0.14 to -0.08$)$, and 0.35 in women (CI -0.38 to -0.33$)$ ), but only for women with unemployment (increase in BMI per 1\% increase in unemployment rate 0.008 (CI -0.008 to 0.02$)$ in men, and 0.036 (CI -0.38 to -0.33 ) in women. Spatial correlations within and between neighbourhoods were small for all of the assessed outcomes.

Conclusions Our findings confirm the previously described association of neighbourhood SES with smoking independent of individual SES, while we found inconsistent associations with SBP and BMI. The neighbourhood environment may be more relevant for behavioural than for biomedical risk factors.

\section{HAVE SOCIO-ECONOMIC DIFFERENCES IN CORONARY RISK FACTORS CHANGED OVER YEARS? RESULTS FROM A POPULATION-BASED STUDY OF MEN BETWEEN 1978-1980 AND 1998-2000}

doi:10.1136/jech.2010.120956.35

${ }^{1} \mathrm{~S}$ E Ramsay, ${ }^{2} \mathrm{P} \mathrm{H}$ Whincu, ${ }^{1} \mathrm{~S}$ L Hardoon, ${ }^{1} \mathrm{M} \mathrm{C}$ Thomas, ${ }^{1} \mathrm{R}$ W Morris, ${ }^{1} \mathrm{~S}$ G Wannamethee. 'Department of Primary Care and Population Health, UCL, London, UK; ${ }^{2}$ Division of Community Health Sciences, St George's University of London, London, $U K$

Background Although CHD mortality has declined in the UK since the late 1970s, the decline has been particularly marked among more affluent subjects. While the decline substantially reflects improvements in established coronary risk factors, little is known about how these have changed in different socio-economic groups.

Objective To examine whether socio-economic differences in coronary risk factors in Britain have changed over 20 years between 1978-80 and 1998-2000.

Design Prospective study of a socio-economically and geographically representative cohort.

Setting 24 British towns.

Participants 4132 men aged $40-59$ years in 1978-80.
Main outcome measures Age-adjusted changes in coronary risk factor levels from 1978-80 to 1998-2000 according to social class were assessed. Coronary risk factors included blood pressure, cholesterol, body mass index (BMI), cigarette smoking and physical activity. Social class, based on longest-held occupation, was grouped as "non-manual" (social classes I, II, III non-manual) and "manual" (III manual, IV and V).

Results Overall, the prevalence of cigarette smoking declined and mean blood pressure and non-HDL cholesterol levels fell, while mean HDL cholesterol and BMI, and physical activity increased. The higher odds of being a current smoker in manual (lower) compared with non-manual (higher) social classes in 2000 (age-adjusted odds ratio 2.04 ; $95 \%$ CI 1.68 to 2.47 ) had not changed since $1978-80$ ( $\mathrm{p}$ for interaction social class*time 0.51 ). Men in manual occupations became less likely to be physically inactive compared with nonmanual groups ( $p$ for interaction 0.04 ) and more likely to be moderate-vigorously active ( $p$ for interaction 0.005). The 20-year increase in mean BMI was $2.34 \mathrm{~kg} / \mathrm{m}^{2}$ in the manual compared with $2.01 \mathrm{~kg} / \mathrm{m}^{2}$ in the non-manual group (difference in mean change $=0.33 \mathrm{~kg} / \mathrm{m}^{2} ; 95 \%$ CI 0.14 to 0.53 ; p for interaction 0.001 ). Mean systolic blood pressure declined more in manual than nonmanual groups (difference in mean change $=3.6$; $95 \%$ CI 2.1 to 5.3 , $p$ for interaction $<0.0001$ ). Non-manual groups had a greater mean decline in non-HDL cholesterol (difference in mean change $=0.18$ $\mathrm{mmol} / \mathrm{l}$; 95\% CI 0.11 to 0.25 , p for interaction $<0.0001$ ) and a greater mean increase in HDL-cholesterol (difference in mean change $0.04 \mathrm{mmol} / 1$; $95 \%$ CI 0.02 to $0.06, \mathrm{p}$ for interaction $<0.0001$ ).

Conclusions Since the 1980s, socio-economic differences in blood pressure and physical activity may have been reduced, while those in cigarette smoking have persisted. Socio-economic differences in BMI, non-HDL and HDL-cholesterol levels appeared to have worsened, with more unfavourable changes in lower socio-economic groups. Continuing priority is needed to improve adverse cardiovascular risk profiles in socially disadvantaged groups in the UK.

\section{Cancer survival}

\section{SOCIO-ECONOMIC INEQUALITIES IN CANCER SURVIVAL IN ENGLAND AFTER THE NHS CANCER PLAN}

doi:10.1136/jech.2010.120956.36

L Ellis, B Rachet, C Maringe, M P Coleman. Epidemiology and Population Health, London School of Hygiene and Tropical Medicine, London, UK

Objectives Socio-economic inequalities in survival have been reported for most adult cancers in England. The NHS Cancer Plan (2000) aimed to improve cancer patient survival, and tackle inequalities in survival between people from deprived and affluent backgrounds. Recent observations suggest some improvements in survival have accelerated since implementation of the Cancer Plan. This study investigates the efficacy of the Cancer Plan in tackling inequalities in cancer survival.

Design We examined data for all adults registered in the National Cancer Registry and diagnosed with one of 21 common cancers in England during 1996-2006 and followed up to 31 December 2007. We defined a priori three calendar periods of diagnosis in relation to the NHS Cancer Plan: 1996-2000 (before the Cancer Plan), 2001-03 (initialisation) and 2004-06 (implementation).

Main outcome measures One-year relative survival by sex and socioeconomic group for each of the 11 calendar years was estimated using a maximum-likelihood approach. Life tables by age, sex, calendar year, deprivation category and Government Office Region were used to control for the variability in background mortality. The "deprivation gap" (quantified as the difference between survival in the most deprived and most affluent groups) was estimated with variance-weighted linear regression. Changes in the deprivation gap 